\title{
An Epidemiological Analysis of Cardiovascular Diseases in Okinawa, Japan
}

\author{
Kunihiko Kinjo, Yorio Kimura, Yuzuru Shinzato, Masayuki Tomori, Yukihiro Komine, \\ Nobuyuki Kawazoe, Shuichi Takishita, Koshiro Fukiyama, and COSMO Group
}

\begin{abstract}
The purpose of the present study is to determine accurate incidence rates of stroke and acute myocardial infarction among the residents in Okinawa, where the census population was $1,222,398$ in 1990 and mortality due to cerebrovascular and heart diseases is lowest in Japan. A co-operative study group including almost all hospitals and clinics in Okinawa was established for the survey. Between April 1, 1988 and March 31, 1991, 4,756 cases of stroke and 1,059cases of acute myocardial infarction were identified. The average age-adjusted annual incidence per 100,000 population was 137 for stroke and 31 for acute myocardial infarction. In the population aged 40 years and older, the respective values were 315 and 72 , indicating the stroke incidence to be 4.4 times higher than that of acute myocardial infarction. The incidence ratio for men to women was 1.7: 1 for stroke and 2.9: 1 for acute myocardial infarction. Among stroke cases, $\mathbf{5 1 . 3 \%}$ were diagnosed as cerebral infarction, $35.7 \%$ as cerebral hemorrhage, 7.7\% as subarachnoid hemorrhage and $5.3 \%$ as others. Computed tomography of the head was performed in $98.4 \%$ of all stroke cases. The case ascertainment, evaluated by comparing the registered cases and death certificates in a certain city, was almost complete. The incidence of acute myocardial infarction is still much lower than that of stroke in Japan. (Hypertens Res 1992; 15: 111-119)
\end{abstract}

Key Words: stroke, myocardial infarction, morbidity, epidemiology

Heart disease and cerebrovascular disease are the second and third leading causes of death in Japan, respectively. Mortality due to stroke has decreased in Japan sharply since the1970's, while the death rate from heart disease has increased, exceeding stroke mortality in $1984(1,2)$. A population-based study in a rural area of northern Japan, however, indicated that in the survey population the 5-year incidence of ischemic heart disease was not increasing and, was much less than that of stroke, which declined by $60 \%$ between 1964-1968 and 1979-1983 (3).

Stroke and ischemic heart disease are leading causes of disability. The proportion of elderly people has increased markedly in Japan. It is predicted that about $24 \%$ of the total population will be comprised of men and women aged 65 years or older in 2020 (1). Owing to this rapid increase in the aged population, cardiovascular disease will inevitably cause even greater disability and death.

Accurate data on cardiovascular disease including the incidence, outcome and risk factors will greatly contribute to the prevention, treatment and development of appropriate health care facilities.

We conducted this epidemiologic study of stroke and acute myocardial infarction on the subtropical islands of Okinawa where the life expectancy of re- sidents is longest and death rates for cardiovascular diseases are lowest in Japan (1).

Okinawa prefecture is located in the southwest part of Japan (Fig. 1). It is composed of more than 100 islands. People inhabit on the 4 main islands and about 35 smaller islands.

As Okinawa is surrounded by the sea and far from the main islands of Japan, patients with stroke or acute myocardial infarction never move from Okinawa, at least in the acute stage, and they are admitted to a regional central hospital which is equipped with modern apparatus including facilities for computed tomography (CT). Patients suffering stroke or acute myocardial infarction on an isolated, small island are usually transferred by aircraft and admitted to a central hospital on a neighboring island. This "self-enclosed" situation of Okinawa facilitates the procurement of accurate data on the incidence of the cardiovascular disease to an extent not possible in other areas in Japan.

\section{Subjects and Methods}

The study population was comprised of all residents in Okinawa. The Census population in 1990 was $1,222,398$ (Fig. 2) and $68 \%$ of all inhabitants were

From the Third Department of Internal Medicine, University of the Ryukyus, School of Medicine, Okinawa, Japan.

This study was supported by Grants of the Ministry of Health and Welfare for 1988, 1989, 1990 (Study on the registration/management/follow-up system of circulatory disease patients), the Grant of Uruma Trust Fund for Research into Science and Humanities for 1989 and the Kimura Foundation Research Grant for 1990. An outline of this study was presented by Prof. K. Fukiyama in his presidential special lecture at the 14th Annual Scientific Meeting of the Japanese Society of Hypertension in Okinawa, 1991.

Address for Reprints: Kunihiko Kinjo, M.D., Third Department of Internal Medicine, University of the Ryukyus, School of Medicine, 207 Uehara, Nishihara-cho, Okinawa 903-01, Japan.

Received and accepted September 4, 1992. 

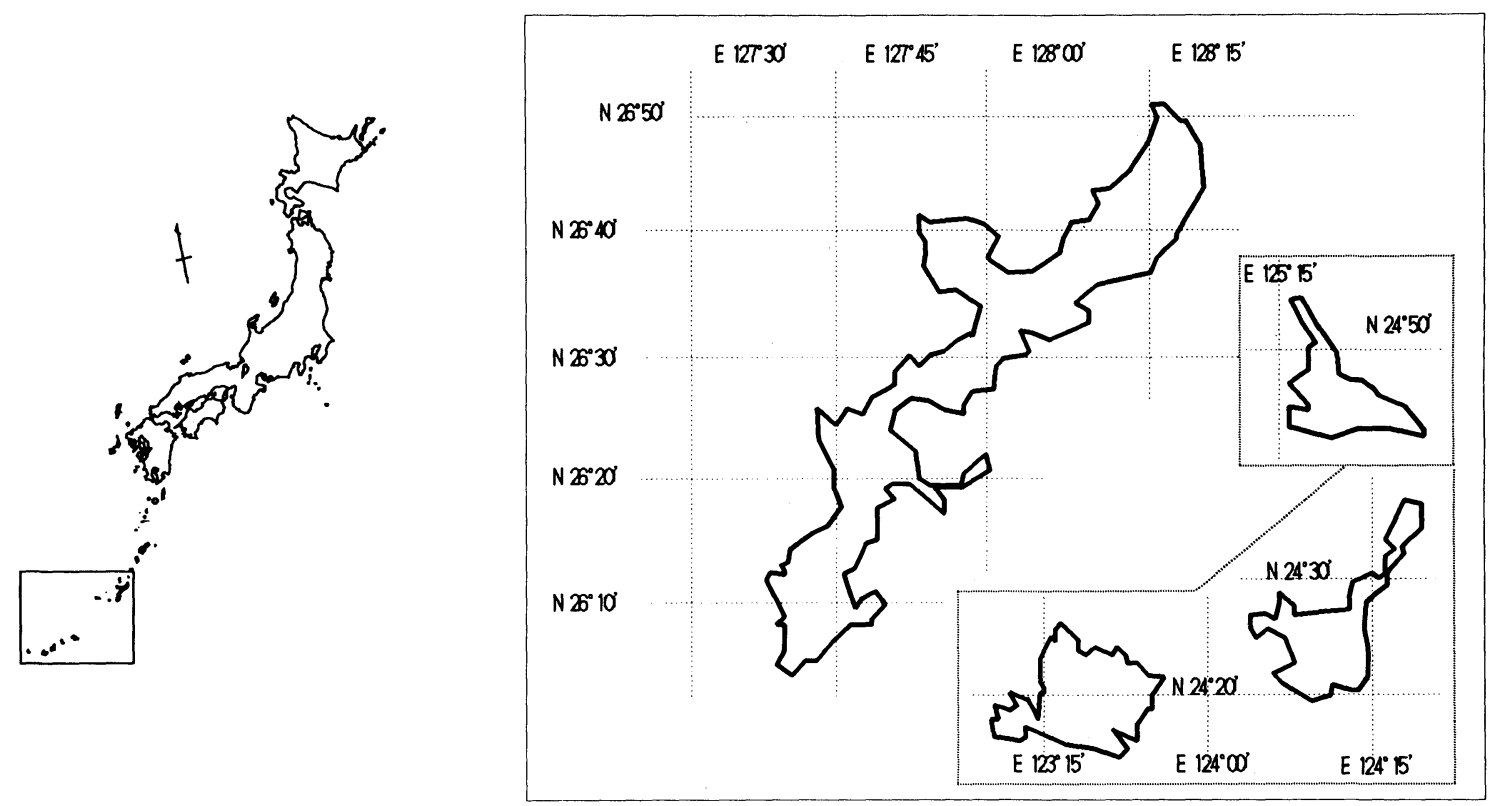

Fig. 1. Okinawa is located in the southwest part of Japan and composed of more than 100 islands. People live on 4 main and about 35 smaller islands.

living in urban areas. Patients who suffered stroke or acute myocardial infarction between April 1, 1988 and March 31, 1991 in Okinawa were registered. Cases with silent myocardial infarction and recurring attacks were included.

Patient registration was achieved through the cooperation of all main hospitals, private hospitals and clinics registered to the Okinawa Medical Association, public health centers and the Environmental and Health Department of the Okinawa Prefectural Government. The study physicians regularly visited these main hospitals where the patients were supposed to be admitted, and investigated medical records, electrocardiograms, laboratory data and CT films. They also visited other medical facilities and carried out similar investigations when informed that the cases had been registered there.

Stroke cases were identified according to the criteria of the Ministry of Health and Welfare (4). Criteria for identification of acute myocardial infarction were similar to those used in the MONICA project (5), and both definite and possible myocardial infarction were included. Death within 30 days after onset was defined as acute death.

\section{Evaluation of Case Ascertainment}

To evaluate the completeness of the case ascertainment, we reviewed death certificates which had been issued during the study period and related to stroke or acute myocardial infarction in a certain city with a population of 41,245 . The death certificate data were compared with the data for the corresponding cases registered in this study.

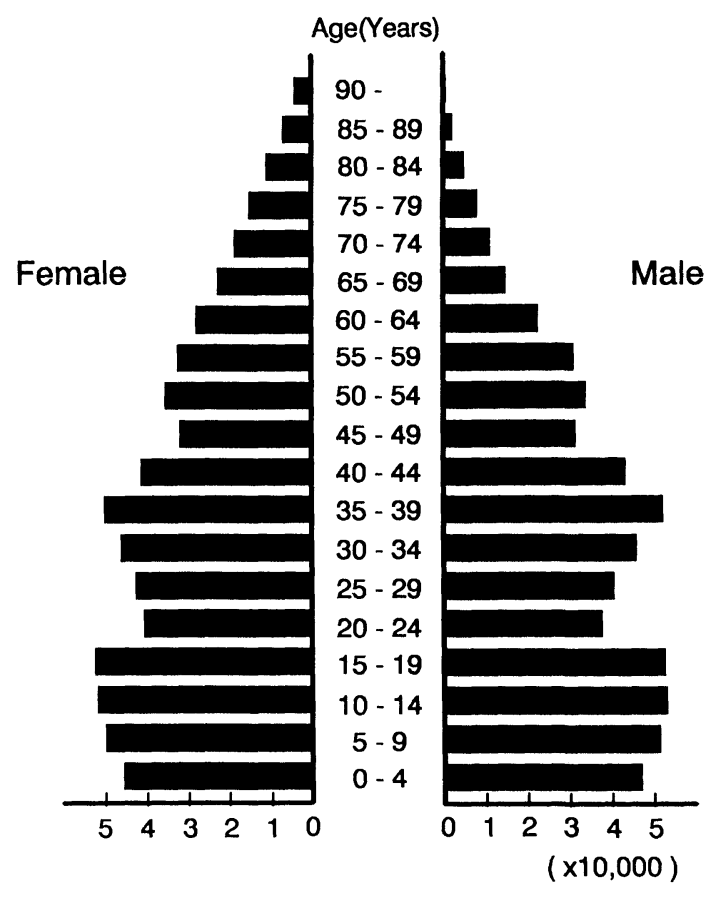

Fig. 2. Age and sex distribution of the residents in Okinawa. Census population of Okinawa was 1,222,398 (598,669 men and 623,729 women) in 1990.

\section{Statistical Analysis}

Crude annual incidence rates were calculated using the census population of Okinawa in 1990 (6). Ageadjusted annual incidence rates were determined by the direct method using the standard population which was derived from the census population of Japan in 1985 (7). Mean ages were expressed as 
mean values $\pm 1 \mathrm{SD}$. The differences of mean age and case-fatality rates between subgroups were examined using normal distribution. The difference of mean age among the subtype of stroke was tested with one-way analysis of variance. Seasonal variations in the occurrence of stroke and acute myocardial infarction were also examined. The distribution of the number of cases was first tested for differences among 3-month intervals of the year with chisquare test for the goodness of fit. If this test showed significant differences, the period with the highest or lowest frequency was tested to evaluate its difference from the average of the other three periods. A $p$ value of less than 0.05 was considered significant.

\section{Results}

Incidence of Stroke and Acute Myocardial Infarction The annual numbers of cases registered were generally constant for both stroke and acute myocardial infarction (Fig. 3).

During the study period, 4,756 cases $(2,573$ men and 2,183 women) of stroke were identified. The age was unknown in three cases, consisting of one male and two females with cerebral infarction. Nine-hundred fifty-nine cases $(20.2 \%)$ were confirmed to be recurrent at the first registration. The average crude annual incidence rate of stroke was 130 per 100,000 population for both sexes (143 for males and 117 for females), and the male: female ratio was 1.2: 1 (Table 1 ). The average age-adjusted annual incidence rate of stroke was 137 for both sexes (180 for males and 105 for females), and the male: female ratio was $1.7: 1$.

In total, 1,059 cases of acute myocardial infarction were registered, of which 695 were male and 364 were female. One-hundred fifty-nine cases $(15.0 \%)$ were recurrent at the first registration. The average crude annual incidence rate of acute myocardial infarction was 29 per 100,000 population for both sexes (39 for males and 20 for females), and

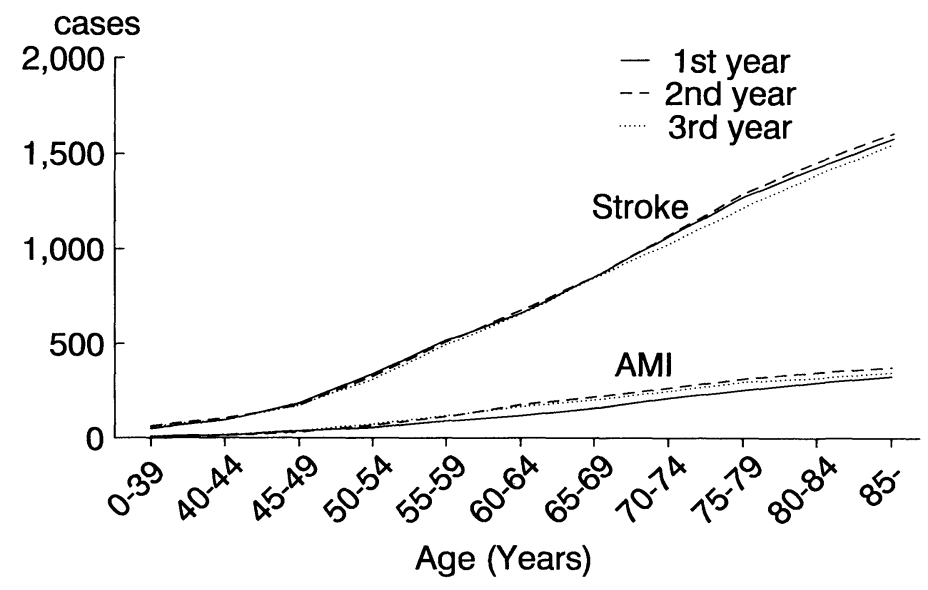

Fig. 3. Cumulative number of cases by age in each year. The numbers of cases with stroke and acute myocardial infarction were 1,584 and 327 in the first year, 1,618 and 381 in the second year and 1,554 and 351 in the third year, respectively. AMI: Acute myocardial infarction.

Table 1. Average Annual Incidence Rate of Stroke and Acute Myocardial Infarction per 100,000 Population

\begin{tabular}{|c|c|c|c|c|c|c|c|c|}
\hline & \multicolumn{4}{|c|}{ Crude } & \multicolumn{4}{|c|}{ Age-adjusted } \\
\hline & Total & Male & Female & $\mathrm{M} / \mathrm{F}$ & Total & Male & Female & $\mathrm{M} / \mathrm{F}$ \\
\hline Stroke & 130 & 143 & 117 & 1.2 & 137 & 180 & 105 & 1.7 \\
\hline cerebral infarction & 66 & 77 & 56 & 1.4 & 73 & 99 & 48 & 2.1 \\
\hline cerebral hemorrhage & 46 & 52 & 41 & 1.3 & 49 & 63 & 38 & 1.7 \\
\hline subarachnoid hemorrhage & 10 & 6 & 13 & 0.5 & 11 & 8 & 14 & 0.6 \\
\hline others & 7 & 8 & 6 & 1.3 & 7 & 10 & 5 & 2.0 \\
\hline Acute myocardial infarction & 29 & 39 & 20 & 2.0 & 31 & 49 & 16 & 3.1 \\
\hline
\end{tabular}

M, Male; F, Female. Census population of Okinawa in 1990: 1, 222, 398 (Male: 598, 669, Female: 623, 729). Standard population: 120, 287, 000. Stroke/Acute myocardial infarction (Age-adjusted): 4.4 (Male: 3.7, Female: 6.6). 
Table 2. Average Annual Incidence Rate of Stroke and Acute Myocardial Infarction per 100,000 Population Aged 40 Years and Older

\begin{tabular}{|c|c|c|c|c|c|c|c|c|}
\hline \multirow{2}{*}{. } & \multicolumn{4}{|c|}{ Crude } & \multicolumn{4}{|c|}{ Age-adjusted } \\
\hline & Total & Male & Female & $\mathrm{M} / \mathrm{F}$ & Total & Male & Female & $\mathrm{M} / \mathrm{F}$ \\
\hline Stroke & 337 & 394 & 289 & 1.4 & 315 & 417 & 241 & 1.7 \\
\hline cerebral infarction & 176 & 217 & 142 & 1.5 & 173 & 233 & 112 & 2.1 \\
\hline cerebral hemorrhage & 119 & 139 & 101 & 1.4 & 113 & 144 & 87 & 1.7 \\
\hline subarachnoid hemorrhage & 24 & 15 & 32 & 0.5 & 24 & 16 & 30 & 0.5 \\
\hline others & 18 & 22 & 14 & 1.6 & 17 & 24 & 12 & 2.0 \\
\hline Acute myocardial infarction & 76 & 107 & 50 & 2.1 & 72 & 113 & 39 & 2.9 \\
\hline
\end{tabular}

Census population aged 40 years and older: 452, 347 (Male: 209, 149 Female: 243, 198). Standard population aged 40 years and older: 50, 412, 000. Stroke/Acute myocardial infarction (Age-adjusted): 4.4 (Male: 3.7, Female: 6.2).

Table 3. Average Annual Age-Specific Incidence Rate per 100,000 Population

\begin{tabular}{crrrrr}
\hline & \multicolumn{2}{c}{ Stroke } & & \multicolumn{2}{c}{ AMI } \\
\cline { 2 - 3 } \cline { 5 - 6 } Age group & Male & Female & & Male & Female \\
\hline $0-29$ & 4 & 2 & & 0 & 0 \\
$30-39$ & 24 & 18 & & 6 & 1 \\
$40-49$ & 97 & 65 & & 36 & 2 \\
$50-59$ & 322 & 173 & & 95 & 15 \\
$60-69$ & 553 & 273 & & 160 & 50 \\
$70-79$ & 1027 & 632 & & 261 & 135 \\
$80-$ & 1319 & 922 & 221 & 185 \\
\hline
\end{tabular}

AMI: Acute myocardial infarction

the male: female ratio was 2.0:1 (Table 1). The average age-adjusted annual incidence rate was 31 for both sexes (49 for males and 16 for females), and the male: female ratio was $3.1: 1$.

As the number of patients with stroke or acute myocardial infarction under the age of 40 years was very small, we calculated the incidence per 100,000 population aged 40 years and older. The average crude annual incidence rate for both sexes was 337 for stroke and 76 for acute myocardial infarction and the ratio of stroke to acute myocardial infarction was 4.4: 1 (Table 2). The average age-adjusted annual incidence rate for both sexes was 315 for stroke and 72 for acute myocardial infarction and the ratio of stroke to acute myocardial infarction was 4.4: 1 (Table 2).

\section{Stroke}

Overall, the occurrence of stroke became prominent from the fifth decade of age, increased steeply with age, and peaked in the eighth decade (Fig. 3). In males, peak occurrence was in the sixth and seventh decades, while it was in the eighth decade in females (Fig. 4). The average annual age-specific incidence rate per 100,000 population increased with age and peaked in the ninth decade and older in both sexes (Table 3). Among the patients with stroke, $51.3 \%$ were diagnosed as cerebral infarction, $35.7 \%$ as cerebral hemorrhage, $7.7 \%$ as subarachnoid hemorrhage and $5.3 \%$ as others, which included undefined types of stroke and transient ischemic attacks. CT was performed in $98.4 \%$ of stroke cases. The average age-adjusted annual incidence rate of cerebral infarction, cerebral hemorrhage, subarachnoid hemorrhage and others were $173,113,24$ and 17 per 100,000 population of age 40 years and older, respectively. The male: female ratios for each subtype of stroke were 2.1 for cerebral infarction, 1.7 for cerebral hemorrhage, 0.5 for subarachnoid hemorrhage and 2.0 for others (Table 2).

The mean age of stroke patients was $66.6 \pm 14.3$ years; it was $64.3 \pm 13.7$ years for males and 69.3 \pm 15.5 years for females and the difference was significant $(p<0.0001)$. The mean ages of the patients with cerebral infarction, cerebral hemorrhage, subarachnoid hemorrhage and others were $69.9 \pm$ $12.8,63.4 \pm 15.0,59.1 \pm 15.2$ and $67.6 \pm 13.6$ years, respectively. The difference among these values was significant $(p<0.001)$. Mean age was lowest in the patients with subarachnoid hemorrhage and highest in those with cerebral infarction. The mean age of females was significantly higher than that of males in all stroke $(p<0.0001)$ as well as in every subtype of stroke, i.e., $72.3 \pm$ 12.4 vs. $67.6 \pm 12.6$ in cerebral infarction $(p<$ $0.0001), 66.9 \pm 15.5 \mathrm{vs} .60 .6 \pm 13.9$ in cerebral hemorrhage $(p<0.0001)$, and $60.9 \pm 15.0$ vs. 55.2 \pm 14.9 in subarachnoid hemorrhage $(p<0.01)$. The ratio of cerebral infarction to cerebral hemorrhage was 1.4: 1 (1.5: 1 for males and 1.4: 1 for females). Under the age of 60 years, cerebral hemorrhage was more common than cerebral infarction. However, cerebral infarction predominated in the seventh decade and older (Table 4).

\section{Acute Myocardial Infarction}

Among 1,059 patients registered, 916 patients were diagnosed as "definite" acute myocardial infarction and 143 patients as "possible" acute myocardial infarction. Electrocardiograms taken at the acute phase could be reevaluated by the study physicians 
Table 4. The Ratio of Cerebral Infarction to Cerebral Hemorrhage

\begin{tabular}{c|c|cc}
\hline Age group & Both sexes & Male & Female \\
\hline $0-29$ & 0.3 & 0.2 & 0.4 \\
$30-39$ & 0.6 & 0.6 & 0.6 \\
$40-49$ & 0.6 & 0.7 & 0.5 \\
$50-59$ & 0.8 & 1.0 & 0.6 \\
$60-69$ & 1.6 & 1.6 & 1.4 \\
$70-79$ & 2.3 & 2.6 & 2.1 \\
$80-$ & 2.1 & 2.7 & 1.8 \\
\hline Total & 1.4 & 1.5 & 1.4 \\
\hline
\end{tabular}

in 938 cases. Six-hundred thirty of these cases $(67.2 \%)$ were $\mathrm{Q}$ wave infarction and $308(32.8 \%)$ were non-Q wave infarction. The infarction site could be determined in 895 cases. Among them, $51.6 \%$ were anterior wall, $41.2 \%$ were lateral wall, and $7.2 \%$ were inferior and/or posterior wall.

The occurrence of acute myocardial infarction became prominent from the fifth decade of age, increased steeply with age and peaked in the sixth and seventh decades in males. In females, it became remarkable from the sixth decade and peaked in the eighth decade (Fig. 4). The mean age of the patients was $66.4 \pm 14.3$ years for both sexes. It was $62.5 \pm 12.7$ years for males and $73.9 \pm 10.9$ years for females, and the difference was significant $(p<$ $0.0001)$. The mean age of all patients with acute myocardial infarction did not differ from that of patients with stroke $(p>0.33)$. The average annual age-specific incidence rate per 100,000 population increased with age and peaked in the eighth decade in males and in the ninth decade and older in females (Table 3).

\section{Acute Death}

Five-hundred eighty-five of 4,756 patients with stroke $(12.3 \%)$ and 242 of 1,059 patients with acute myocardial infarction $(22.9 \%)$ died within 30 days after onset. The mean ages of cases suffering acute death were $67.3 \pm 16.4$ years old $(64.0 \pm 16.1$ in males, $70.8 \pm 16.1$ in females) in stroke and 73.2 \pm 11.9 years old $(69.2 \pm 12.0$ in males, $77.9 \pm$ 10.0 in females) in acute myocardial infarction. Mean age differed significantly between the cases with stroke and acute myocardial infarction $(p<$ 0.0001 ) and between males and females for both diseases $(p<0.0001)$ and among the subtypes of stroke, i.e., $74.5 \pm 11.7 v s .78 .3 \pm 12.2$ in cerebral infarction $(p<0.05), 69.3 \pm 16.8$ vs. $59.9 \pm 15.9$ in cerebral hemorrhage $(p<0.0001)$, and $65.4 \pm 15.4$ $v s .58 .2 \pm 14.7$ in subarachnoid hemorrhage $(p<$ $0.01)$.

The case-fatality rates were significantly different between stroke and acute myocardial infarction ( $p$ $<0.0001$, Table 5). The case-fatality rate was significantly higher in females than in males in acute myocardial infarction $(p<0.0001)$. On the contrary, it was higher in males than in females in cerebral hemorrhage $(p<0.05)$. There were no significant sex-related differences in case-fatality rates in all stroke cases, cerebral infarction and subarachnoid hemorrhage $(p>0.1)$. Among the subtypes of stroke, the case-fatality rate was highest in subarachnoid hemorrhage.

\section{Seasonal Variations}

The occurrence of cerebral hemorrhage showed seasonal variations $(p<0.001)$ : a peak in winter and a trough in summer. However, no significant seasonal differences were found in the other types of stroke and in total stroke cases $(p>0.05$, Fig. 5). Seasonal variations were likewise not found in the occurrence of acute myocardial infarction $(p>0.05)$.

\section{Case Ascertainment}

Eighty-seven death certificates relating to stroke or acute myocardial infarction were identified in the designated city and 85 of them had also been registered in the present study (97.7\%, Table 6). There were 31 death certificates in which the cause of death had been diagnosed as so-called "acute heart failure" or "acute cardiopulmonary insufficiency."

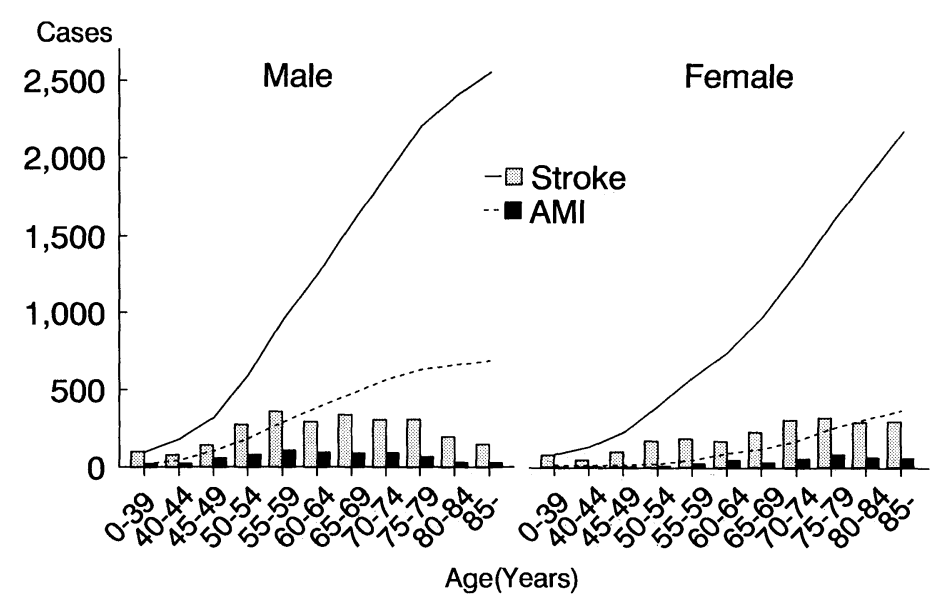

Fig. 4. Cumulative number of the cases for 3 years by sex: In males, the numbers of the cases with stroke and acute myocardial infarction were 2,573 and 695, respectively. They were 2,183 and 364 in females. AMI: Acute myocardial infarction. 
Table 5. Death within 30 Days after the Onset and the Case-Fatality Rate

\begin{tabular}{lcrr}
\hline & Total $(\%)$ & Male $(\%)$ & Female $(\%)$ \\
\hline Stroke & $585(12.3)$ & $314(12.2)$ & $271(12.4)$ \\
cerebral infarction & $159(6.5)$ & $89(6.4)$ & $70(6.7)$ \\
cerebral hemorrhage & $294(17.3)$ & $177(19.0)^{*}$ & $117(15.2)$ \\
subarachnoid hemorrhage & $120(32.7)$ & $43(37.1)$ & $77(30.7)$ \\
others & $12(4.8)$ & $5(3.5)$ & $7(6.4)$ \\
Acute myocardial infarction & $242(22.9)^{* *}$ & $129(18.6)^{* *}$ & $113(31.0)^{* * \#}$ \\
\hline
\end{tabular}

${ }^{*} p<0.05$ vs. female. ${ }^{* *} p<0.0001$ vs. stroke. ${ }^{\#} p<0.0001$ vs. male.

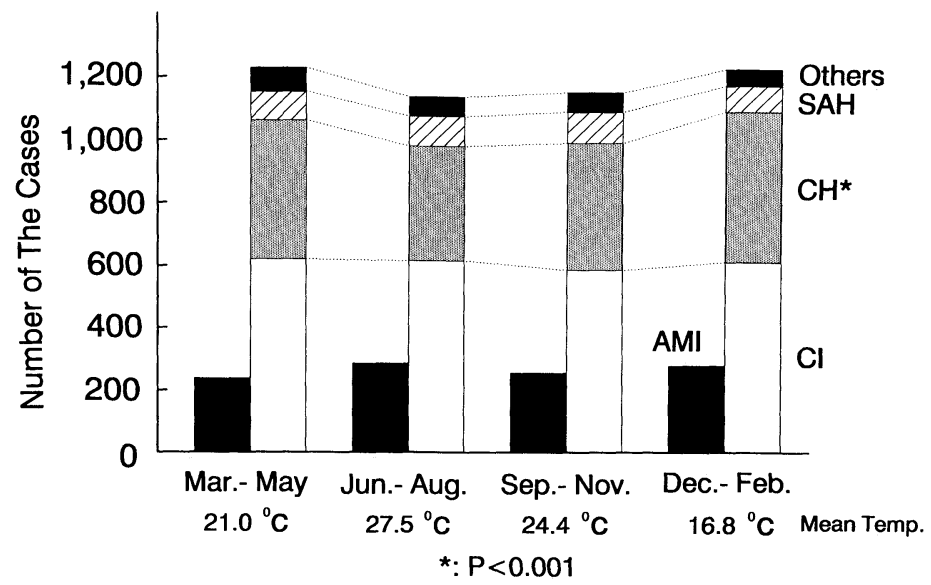

Fig. 5. Seasonal variations of stroke and acute myocardial infarction form April 1, 1988 to March 31, 1991 in Okinawa. There were no significant seasonal variations in occurrence for total stroke and acute myocardial infarction. However, the occurrence of cerebral hemorrhage decreased in summer and increased in winter significantly (p <0.05). AMI: Acute myocardial infarction. CI: Cerebral infarction. CH: Cerebral hemorrhage. SAH: Subarachnoid hemorrhage.

Table 6. Evaluation of the Completeness of Case Ascertainment by Death Certificates in a Certain city. The Numbers of Death Certificates Issued during the Study Period and the Corresponding Nunbers of Registered and Unregistered Cases in the Present Study Are Shown

\begin{tabular}{lccc}
\hline & Death & Registered & Unregistered \\
cartificates & cases & 2 \\
\hline Stroke & 63 & 61 & 2 \\
cerebral infarction & 28 & 26 & 0 \\
cerebral hemorrhage & 26 & 26 & 0 \\
subarachnoid hemorrhage & 8 & 8 & 0 \\
others & 1 & 1 & 0 \\
Acute myocardial infarction & 24 & 24 & \\
\hline
\end{tabular}

Such cases either died within a few hours after onset or were found dead. As they lacked records such as accurate case histories, electrocardiograms and laboratory data, they were not included in the present study.

\section{Discussion}

To be meaningful, the incidence of cardiovascular disease should be established through an epidemiologic study based on complete case ascertainment and high diagnostic accuracy in a sufficiently large population which includes all age-groups, both genders, various occupations and both urban and rural areas. We perceived that patients in Okinawa with acute cardiovascular diseases such as acute myocardial infarction or stroke were never referred to a hospital outside of Okinawa. In addition, this study was performed with the cooperation of all main hospitals and other smaller hospitals and clinics through the Okinawa Medical Association. To identify patients with acute episodes of cardiovascular disease who had been treated at home without visiting a clinic or hospital, we also enlisted the cooperation of public health centers. Since virtually all medical facilities in Okinawa participated in the 
present study, almost all cases of stroke and acute myocardial infarction occurring during the study period were assumed to be identified. The evaluation of case ascertainment supports this supposition.

Diagnosis was made according to the criteria used in the MONICA Project for acute myocardial infarction and the criteria of the Ministry of Health and Welfare for stroke $(4,5)$. All diagnoses were reviewed by several doctors who were fully familiar with the criteria. CT had been performed in $98.4 \%$ of the stroke cases and was utilized to classify stroke into subtypes. Therefore, despite the large population, almost complete case ascertainment and diagnostic accuracy were considered to be achieved in the present study.

Although there may be some differences in the age distribution of the study population, methods of case ascertainment and diagnostic criteria for stroke and acute myocardial infarction, it would be of epidemiologic interest to compare the annual incidence of stroke and acute myocardial infarction and the ratio of these diseases in Okinawa with the respective values in Hisayama, Akita in the northern part of Japan, Honolulu and Framingham (8-13). The Hisayama, Honolulu, and Framingham studies were cohort studies and did not include recurrent cases. In the Honolulu study, mortality from coronary heart disease was included along with acute myocardial infarction, and only Japanese male cases were investigated. The age of the population at the beginning of the study was 40-64 years old in the Hisayama study, 45-68 years old in the Honolulu study and 30-59 years old in the Framingham study and the follow-up periods were 20 years, 6 years and 24 years, respectively. The study performed in Akita was a cross-sectional study and included recurrent cases, but investigated only stroke cases which underwent $\mathrm{CT}$.

If confirmed recurrent cases are excluded, the average crude annual incidence rate of stroke in males in Okinawa becomes 3.1 per 1,000 population aged 40 years and older, compared with 8.1 personyears per 1,000 in Hisayama, 3.2 per 1,000 population aged between 40 to 79 years in Akita, 2.8 person-years in Honolulu and 2.6 person-years in Framingham, respectively. Similarly, the average crude annual incidence rates of acute myocardial infarction in men were 0.9 in Okinawa, 2.1 in Hisayama, 3.0 in Honolulu and 5.5 in Framingham.

These comparisons indicate that the incidence rate of acute myocardial infarction is lowest in Okinawa and highest in Framingham among the four regions studied. The incidence rate of stroke in Okinawa seemed to be lower than that in Hisayama and Akita, but still higher than that in Honolulu and Framingham.

The reasons behind the relatively low incidence of cardiovascular disease in Okinawa remain unknown. Some people may attribute it to the relatively low salt intake of residents in Okinawa compared with those who live in other areas in Japan. According to recent nutrition surveys performed in Japan, the Japanese average salt intake is about $12 \mathrm{~g}$ per day, but it is only about $10 \mathrm{~g}$ per day for residents of Okinawa (14). However, in the population aged 15 years and older, the proportion of the people with hypertension or borderline hypertension is $41 \%$ in Okinawa and $37 \%$ in Japan as a whole $(1,14)$, and the difference is not statistically significant $(p>0.1)$. Mild climate may possibly contribute to the low incidence of cardiovascular disease in Okinawa. Some studies have shown a significant relationship between low temperature and the occurrence of stroke $(10,15)$.

The proportion of cerebral hemorrhage in men with stroke was about $36.0 \%$ in Okinawa, $24.7 \%$ in Hisayama, $31.2 \%$ in Akita, $14.3 \%$ in Honolulu and $7.7 \%$ in Framingham, respectively. The reasons for the highest relative frequency of cerebral hemorrhage in Okinawa among the five regions are also not known. This may be partly due to a high rate of performing CT in the present study. CT can reveal small hematomas that might be misdiagnosed as cerebral infarction by clinical judgement. According to previous studies which compared clinical diagnosis with autopsy or findings of computed tomography of the head, a correct clinical diagnosis was made in $65-86 \%$ of cerebral hemorrhage, $64-85 \%$ of cerebral infarction and $68-90 \%$ of subarachnoid hemorrhage cases $(10,16,17)$. Some people may suggest that the high proportion of cerebral hemorrhage and high rate of performing CT in the present study reflects the poor identification of cerebral infarction with mild symptoms. However, this suspicion is off the point because the case-fatality rate within 30 days after onset was not high but rather low in comparison with previous studies $(18-20)$ and case ascertainment was estimated to be almost complete. Poor management of hypertension may be one of the reasons for the high proportion of cerebral hemorrhage in Okinawa.

Subarachnoid hemorrhage occurred predominantly in women as in other studies $(9,20,21)$. However, the proportion of subarachnoid hemorrhage in the present study was lower than in other previous studies in Japan $(10,16,17,22)$ and almost similar to those in the United States and in France (19, $23)$. Some previous studies showed that more than $75 \%$ of subarachnoid hemorrhage cases occurred in the age group under 60 years old $(24,25)$. However, similar to the study in Akita prefecture (53.9\%) in Japan (10), it was only $52.9 \%$ in the present study.

Although no seasonal variations in the occurrence of total stroke cases and acute myocardial infarction cases were found, the present study showed that even in subtropical areas, cerebral hemorrhage was associated with significant seasonal variations. Previous reports in Australia and Akita prefecture showed similar trends, i.e., a high incidence of cerebral hemorrhage in winter and a low incidence in summer $(10,15)$. This phenomenon may suggest that even mildly. low temperatures have a greater influence than previously suspected on the occurrence of cerebral hemorrhage.

A significantly higher case-fatality rate in women than in men within 30 days after onset was found for acute myocardial infarction. This may partly be 
attributed to the significantly higher mean age of women than men, differing by about 11 years. However, the case-fatality rate in the acute phase in Okinawa is still lower than in previous reports (26, 27).

The present study showed that the incidence of acute myocardial infarction is still much lower than that of stroke in Japan and suggests that the low incidence of cardiovascular disease may be one of major reasons for the longevity of the residents in Okinawa. Further investigations are needed for indepth analyses of risk factors and factors influencing patient prognosis. Such studies should greatly contribute to understanding of the mechanism, prevention and treatment of cardiovascular disease.

\section{References}

1. Health and Welfare Statistics Association: Health services in Japan (Kokumin eisei no doko). Indices of Health and Welfare (Kousei no shihyo) 1990; 37: 402, 424 (Suppl).

2. Hirota Y: Epidemiology of stroke. Brain and Nerve (Nousinkei) 1982; 34: 437-449.

3. Shimamoto $\mathrm{T}$, Komachi $\mathrm{Y}$, Inada $\mathrm{H}$, et al: Trends for coronary heart disease and their risk factors in Japan. Circulation 1989; 79: 503-515.

4. Tazaki Y: Topics of cerebrovascular disturbance. Medicina 1984; 21: 1897-1899.

5. WHO MONICA Project: WHO MONICA Project: Assessing CHD mortality and morbidity. Int $J$ Epidemiol 1989; 18 (Suppl 1): S38-S45.

6. Statistics Bureau Management and Coordination Agency: 1990 Population Census of Japan. Vol. 2, Part 2, 47 Okinawa-ken. 1991.

7. Health and Welfare Statistics Association: Health services in Japan (Kokumin eisei no doko). Indices of Health and Welfare (Kousei no shihyo) 1991; 38: 12-17.

8. Ueda K: Myocardial infarction in Hisayama. Diagnosis and Treatment (Shindan to chiryo) 1986; 74: 1585-1588.

9. Kikumura T: Risk factors for cerebral infarction. A prospective clinico-epidemiological study in Hisayama town, Japan, and a comparison with the survey findings from Framingham, Massachusetts, USA Fukuoka Acta Medica 1985; 76: 343-364.

10. Suzuki K, Kutsuzawa T, Takita K, et al: Clinicoepidemiologic study of stroke in Akita, Japan. Stroke 1987; 18: 402-406.

11. Kagan A, Popper JS, Rhoads GG: Factors related to stroke incidence in Hawaii Japanese men. The Honolulu Heart Study. Stroke 1980; 11: 14-21.

12. Robertson TL, Kato H, Rhoads GG, et al: Epidemiologic studies of coronary heart disease and stroke in Japanese men living in Japan, Hawaii and California. Incidence of myocardial infarction and death from coronary heart disease. Am J Cardiol 1977; 39: 239-243.

13. Dawber TR: The Framingham study. The epidemiology of atherosclerotic disease. Cambridge, Mass, Harvard University Press, 1980, pp59-75.

14. Environmental and Health Department of Okinawa Prefectural Government: The 1988 Nutrition Survey of the residents in Okinawa Prefecture (Kenmin eiyo no genjyo). Environmental and Health Department of Okinawa Prefectural Government, Okinawa, 1990.

15. Christie D: Stroke in Melbourne, Australia: An epidemiological study. Stroke 1981; 12: 467-469.
16. Katsuki S: Hisayama study. J Jpn Soc Inter Med 1971; 60: 3-17.

17. Tanaka $\mathrm{H}$, Ueda $\mathrm{Y}$, Date $\mathrm{C}$, et al: Incidence of stroke in Shibata, Japan: 1976-1978. Stroke 1981; 12: 460-466.

18. Boysen G, Nyboe J, Appleyard M, et al: Stroke incidence and risk factors for stroke in Copenhagen, Denmark. Stroke 1988; 19: 1345-1353.

19. Giroud M, Beuriat P, Vion P, D'Athis PH, Dusserre L, Dumas R: Stroke in a French prospective population study. Neuroepidemiology 1989; 8: 97-104.

20. Ricci S, Celani MG, Guercini G, et al: First-year results of a community-based study of stroke incidence in Umbria, Italy. Stroke 1989; 20: 853-857.

21. Haberman S, Capildeo R, Rose FC: Sex differences in the incidence of cerebrovascular disease. $J$ Epidemiol Community Health 1981; 35: 45-50

22. Kiyohara Y, Ueda K, Hasuo Y, et al: Incidence and prognosis of subarachnoid hemorrhage in a Japanese rural community. Stroke 1989; 20: 1150-1155

23. Broderick JP, Phillips SJ, Whisnant JP, O'Fallon WM, Bergstralh EJ: Incidence rates of stroke in the eighties: The end of decline in stroke? Stroke 1989; 20: $577-582$

24. Nishimoto A, Ueta $\mathrm{K}$, Onbe $\mathrm{H}$, et al. Nationwide cooperative study of intracranial aneurysm surgery in Japan. Stroke 1985; 16: 48-52

25. Yoshimoto T, Kayama T, Kodama N, Suzuki J: Distribution of intracranial aneurysm, in Suzuki J (ed): Cerebral aneurysms. Neuron Publ Co, 1979, pp1419.

26. Beaglehole R, Dobson A, Hobbs M, et al: Comparison of event rates among three MONICA Centers. Acta Med Scand 1988; Suppl 728: 53-59.

27. Dobson AJ, Alexander HM, Al-Roomi K, et al: Coronary events in the Hunter region of New South Wales, Australia: 1984-1986. Acta Med Scand 1988; Suppl 728: 84-89.

\section{APPENDIX}

Co-operative Study Group of Morbidity and Mortality of Cardiovascular Diseases in Okinawa (COSMO Group)

University of The Ryukyus

Third Department of Internal Medicine K. Fukiyama

(Chairman), K. Kinjo, Y. Kimura, Y. Shinzato,

M. Tomori, Y. Komine, N. Kawazoe, S. Takishita,

Y. Maruoka, K. Iseki, S. Kaseda, H. Muratani,

T. Matsumoto, M. Tozawa, T. Sugama, T. Touma

Second Department of Internal Medicine

R. Sunagawa, F. Nagamine

Second Department of Surgery K. Iha

Department of Neurosurgery T. Kinjo

Department of Legal Medicine H. Nagamori,

Y. Ohno, T. Mukai

Department of Community Service M. Suzuki,

M. Adachi

Okinawa Prefectural Chubu Hospital J. Arakaki,

H. Asato, K. Toyonaga, T. Nishihira, K. Kaneshiro, M. Kyushimsa, S. Toma

Okinawa Prefectural Naha Hospital M. Yamauchi,

Y. Oshiro, K. Kakazu, K. Kuniyoshi

Okinawa Prefectural Nanbu Hospital K. Kinjo, M. Arakaki, N. Uechi

Okinawa Prefectural Nago Hospital H. Ishijima,

A. Tokuda, Y. Ikema

Okinawa Prefectural Yaeyama Hospital N. Ohama, Y. Ishigaki, M. Imamura

Okinawa Prefectural Miyako Hospital H. Takaesu,

T. Wake, N. Onaga

Naha City Hospital T. Tabata, S. Sakumoto 
Okinawa Red Cross Hospital M. Higa, E. Takara, M. Toguchi, T. Morita

Izumizaki Hospital Y. Shiroma, T. Kikumura Makiminato Chuo Hospital E. Yamaguchi, H. Ikeda, K. Higa

Nakagami Hospital C. Oyama, K. Nakada, M. Ishihara Okinawa Kyodo Hospital T. Nakanishi, J. Moromikawa Nanbu Tokushukai Hospital E. Henzan, T. Kadekaru, N. Gushiken, M. Yamaguchi

Chubu Tokushukai Hospital Y. Horikawa, M. Nishihira Minei Hospital S. Minei

Kitagami Chuo Hospital A. Hokama, N. Kinjo

Kaiho Hospital T. Funakoshi, T. Hidaka

Urasoe General Hospital T. Miyagi, K. Tokuyama

Ginowan Memorial Hospital Y. Kinjo, D. Kitagawa,

A. Morishima

Okinawa Medical Hospital T. Maeshiro, K. Kadena, J. Nakamasu

Noge Hospital Y. Ueta

Yonabaru Chuo Hospital K. Nakao, H. Yogi

Chuzan Hospital H. Suenaga, Y. Toda

Tomishiro Chuoh Hospital K. Higa, S. Higa, T. Shinjo Ohhama Daiichi Hospital H. Ohhama, M. Nakamoto Ohhama Daini Hospital S. Sugama, M. Uezu Kinjo et al.
Okinawa Daiichi Hospital N. Miyagi

Ryusei Hospital T. Oshiro, K. Nakasone

Okinawa Central Hospital Y. Ohnaka

NaKa Hospital T. Naka

Yokatsu Hospital M. Shiohira

Motobu Memorial Hospital T. Takaishi

Heart Life Hospital I. Tengan, T. Asato

The Okinawa Medical Association

The Environmental and Health Department

of the Okinawa Prefectural Government M. Zentoku

Okinawa Prefectural Chuo Public Health Center

K. Sunagawa

Okinawa Prefectural Nanbu Public Health Center

H. Hirata

Okinawa Prefectural Koza Public Health Center

T. Inami

Okinawa Prefectural Ishikawa Public Health Center

K. Kishimoto

Okinawa Prefectural Nago Public Health Center

T. Shimabukuro

Okinawa Prefectural Yaeyama Public Health Center T. Aoyama

Okinawa Prefectural Miyako Public Health Center M. Higa 